\title{
Pratiques
}

Linguistique, littérature, didactique

191-192 | 2021

Théâtre contemporain : Écritures et Représentations

\section{É. Louis et D. Eribon au théâtre : Performer le réel et documenter l'intime. L'autosociobiographie en scène}

É. Louis and D. Eribon in the theater: Performing the real and documenting the intimate. Autosociobiography on stage

\section{Delphine Edy}

\section{OpenEdition \\ Journals}

Édition électronique

URL : https://journals.openedition.org/pratiques/11257

DOI : $10.4000 /$ pratiques. 11257

ISSN : 2425-2042

Éditeur

Centre de recherche sur les médiations (CREM)

\section{Référence électronique}

Delphine Edy, «É. Louis et D. Eribon au théâtre : Performer le réel et documenter l'intime.

L'autosociobiographie en scène », Pratiques [En ligne], 191-192 | 2021, mis en ligne le 15 décembre

2021, consulté le 04 janvier 2022. URL : http://journals.openedition.org/pratiques/11257 ; DOI :

https://doi.org/10.4000/pratiques. 11257

Ce document a été généré automatiquement le 4 janvier 2022.

(C) Tous droits réservés 


\title{
É. Louis et D. Eribon au théâtre : Performer le réel et documenter l'intime. L'autosociobiographie en scène
}

\author{
É. Louis and D. Eribon in the theater: Performing the real and documenting the \\ intimate. Autosociobiography on stage
}

Delphine Edy

\section{Introduction}

1 Les noms de D. Eribon et d'É. Louis sont aujourd'hui bien connus du grand public. Depuis la parution d'En finir avec Eddy Bellegueule en 2014, son premier récit autobiographique, É. Louis enchaine les succès en librairie : en creusant le sillon de son histoire personnelle afin de cartographier le monde social et la violence et la domination qui y règnent, il écrit « pour les invisibles mais aussi pour ses ennemis en les confrontant au monde qu'ils ne veulent pas voir » (Louis, 2021b).

2 D. Eribon, sociologue et philosophe, a plus d'une vingtaine d'ouvrages à son actif. Mais c'est avec Retour à Reims que l'on associe le plus souvent son nom. Publié en 2009 et vendu à plus de 65000 exemplaires en l'espace de six ans, alors qu'« à 1300 , on estime souvent qu'un livre de sciences sociales est déjà un succès correct » (Leprince, 2019), le livre a été immédiatement un succès éditorial et s'est vu réédité deux fois, en 2010 et en 2018.

Jusqu'en 2009, Eribon, qui est venu à la sociologie après la philosophie, avait plutôt travaillé sur le genre et la sexualité. Avec Retour à Reims, il s'attaque aux trajectoires ascendantes depuis son cas personnel. Il tente une forme d'ethnographie de l'intime pas toujours expurgée d'affects, et tissée de références qui moissonnent aussi bien du côté de la littérature, de la philosophie, que de la sociologie (Id.). 
En faisant le choix de l'écriture à la première personne, D. Eribon, ne propose « pas un livre de sociologie à proprement parler. C'est un livre personnel dans lequel il revient sur une partie de sa vie qu'il a occultée durant de longues années »(Ladouceur, 2010). La réception par la presse généraliste est unanime et enthousiaste. J.-L. Jeannelle (Le Monde) salue un «essai où [Eribon] emprunte à la tradition littéraire du récit de retour ", en racontant "l'histoire de vies qui se sont croisées sans vraiment s'être rencontrées » (Jeannelle, 2009). Jérôme Duuis (L'Express) y voit « le formidable comingout social » d'un D. Eribon (2009, p. 107) « sociologue de sa propre autobiographie qui livre un véritable "roman familial des origines ». Pour C. Ferniot (Télérama), Retour à Reims s'entend comme un «beau récit tendu mêl[ant] la réflexion intellectuelle sur l'identité et l'histoire singulière et intime » (Ferniot, 2009, p. 69) :

À l'autobiographie, le philosophe D. Eribon a préféré une autre forme littéraire qui rappelle celle d'Annie Ernaux, souvent citée. Elle lui permet d'exprimer les tâtonnements d'une vie et montre les batailles permanentes d'un homme dominé par la violence d'une société de classes (id.).

4 "Récit de retour ", " histoire de vie ", « autobiographie ", « roman familial », tous ces termes mettent en lumière la construction d'une forme littéraire, et l'on saisit mieux ce que dit A. Ernaux (2009, p. 118) de l'écriture en creux dans Retour à Reims : «Eribon offre ici un exemple magnifique de la vie éclaircie [...] dans une démarche d'écriture qui lie étroitement l'intime, le social et le politique, unit le corps usé d'une mère à la division injuste d'une société à changer ».

5 Au-delà de leurs succès éditoriaux, les récits autosociobiographiques ${ }^{1}$ de D. Eribon et d'É. Louis s'imposent également depuis quelques années sur les scènes contemporaines européennes. En février 2015, L. Hatat, le fondateur et directeur artistique de la compagnie anima motrix qui aime mettre en lumière la violence des mécanismes de domination à travers des textes classiques ou contemporains, proposait la première mise en scène de Retour à Reims à la Maison des Métallos dans le $20^{\text {e }}$ arrondissement de Paris. S'en est suivie une longue tournée française jusqu'à une reprise en Avignon en juillet 2017 au Théâtre des lucioles. En 2020, il adaptait et comettait en scène avec E. Gustafsson Histoire de la violence d'É. Louis que l'on pouvait encore voir en juillet 2021 au Festival Off d'Avignon.

6 En 2017, T. Ostermeier, le directeur artistique de la Schaubühne à Berlin proposait avec Return to Reims un spectacle hybride entre film documentaire et performance théâtrale et musicale, qui allait par la suite se démultiplier en différentes langues. Créé en anglais à Manchester en juillet 2017 dans le cadre du Manchester International Festival, repris l'année suivante en allemand à la Schaubühne, puis en français début 2019 au Théâtre de la Ville à Paris, c'est le Piccolo Teatro de Milan qui accueillait ensuite, en novembre 2019, la version italienne Ritorno a Reims, alors qu'une nouvelle version allemande (avec une nouvelle distribution) figure au répertoire de la Schaubühne depuis la première qui a eu lieu le 30 juin 2021 et que la première d'une version suédoise, Tillbaka till Reims, a eu lieu le 26 septembre dernier.

7 Parallèlement aux versions multilingues de Retour à Reims, T. Ostermeier choisissait également de monter deux textes d'É. Louis. Avec Au cœur de la violence (Louis \& Ostermeier, 2019), une version réécrite en allemand par l'auteur, le metteur en scène et le dramaturge F. Borchmeyer, dont la première a eu lieu le 3 juin 2018 à Berlin, il signe une mise en scène qui connait depuis un écho international retentissant malgré la crise sanitaire. Le spectacle, au répertoire de la Schaubühne, s'est notamment joué en Grèce à 
guichets fermés au festival d'Épidaure les 5 et 6 octobre derniers. Par ailleurs, le troisième livre d'É. Louis a trouvé une actualisation scénique inattendue dans une nouvelle collaboration entre l'auteur et $\mathrm{T}$. Ostermeier - dont la première aurait dû avoir lieu le 20 mars 2020 à Berlin dans le cadre du Festival FIND² - qui ont présenté à Berlin Qui a tué mon père de et par $^{3}$ É. Louis le 7 octobre $2021^{4}$, spectacle que les spectateurs parisiens ${ }^{5}$ ont pu voir au Théâtre de la ville lors d'une séance de travail le 15 février puis en septembre 2020. Dans ce spectacle, É. Louis, en plus d'être auteur, devient acteur, et la double équation " personnage = acteur = personne $»$ interroge de plain-pied les rapports entre narration, théâtre, réel et performance.

8 Ce troisième texte d'É. Louis a par ailleurs ceci de spécifique qu'il a été pensé et écrit pour la scène : c'est en effet au cours d'un échange avec S. Nordey, acteur, metteur en scène et directeur du Théâtre national de Strasbourg (TNS), que l'idée est née.

C'était après une lecture publique d'Histoire de la violence, son deuxième roman, à Strasbourg. Nous dinions avec l'auteur allemand Falk Richter. C'était arrosé, fraternel. À la fin du repas, j'ai lancé à Édouard: "Si tu as envie d'écrire pour le théâtre, sache que les portes du TNS te sont ouvertes ». Un an plus tard, je reçois un mail avec le texte en pièce jointe (Nordey, 2020).

9 Immédiatement, il a été décidé que S. Nordey interpréterait le rôle d'Édouard : "Je trouvais ce monologue formidable, mais je ne le pensais pas pour moi. Je n'ai pas l'âge du fils, je ne me sentais pas légitime. Je suis un vieux croûton! Lui était catégorique. Donc j'ai accepté, avec avidité et inquiétude »(Id.). S. Nordey mettra par ailleurs en scène en 2022 dans le cadre du Printemps des Comédiens de Montpellier le deuxième volet du diptyque, Combats et métamorphoses d'une femme (paru en 2021).

Enfin, I. van Hove, le directeur artistique de l'International Theater Amsterdam, bien connu des spectateurs français pour avoir signé plusieurs spectacles mémorables, notamment Les Damnés dans la Cour d'honneur à Avignon (2016) ou sa version d'Euripide Électre/Oreste à la Comédie-Française (2019), a lui aussi choisi de s'emparer du diptyque d'É. Louis que forment Qui a tué mon père et Combats et métamorphoses d'une femme : à l'été 2020 , il proposait une version en néerlandais très remarquée par la critique de Qui a tué mon père avec le grand acteur H. Kesting dans le rôle-titre, puis, le 9 septembre dernier, Combats et Métamorphoses d'une femme, avec M. Heebink dans le rôle de la mère et $\mathrm{M}$. Mardo dans le rôle d'Édouard.

11 Si l'on en croit l'engouement de ces grands metteurs en scène européens pour ces textes, il apparait que l'écriture de soi implique une dimension scénique et performative propre à attirer l'attention des gens de théâtre, peut-être particulièrement sensibles à la qualité des écritures en je(u) dans le travail de ces auteurs. Recréés pour la scène, ces textes permettent visiblement de dépasser les dichotomies réalité/fiction, texte/performance et texte narratif/texte dramatique. Il s'agira donc dans cette contribution de réfléchir à cette "pratique dramatico-théâtroperformative [qui] ne cesse d'ouvrir les pièces à des pans du réel »(Pavis, 2018, p. 369), en s'appuyant sur Retour à Reims et Qui a tué mon père, et de proposer un cadre pour penser ces textes transpersonnels, devenant des performances transmédiales où la littérature rencontre les arts, à la croisée d'enjeux esthétiques et politiques. 


\section{Un « je transpersonnel »}

12 Les textes de D. Eribon et d'É. Louis sont des récits à la première personne, dans lesquels il y a une identification assumée entre l'auteur et le narrateur, c'est de leur propre je dont il est question et non d'un autre : le « contrat de lecture » (Lejeune, 1996, p. 44) est clair. C'est d'ailleurs ce pacte de vérité qui leur permet d'aller à la recherche du réel. En choisissant d'emprunter explicitement les traces d'A. Ernaux, D. Eribon écrit lui-aussi, vingt ans après Une Femme, « quelque part entre la littérature, la sociologie et l'histoire » (Ernaux, 1988, p. 23), là où É. Louis n'écrit pas seulement « au-dessous de la littérature " (Id.) mais délibérément «contre» elle (Louis, 2021a, p. 20). Quand A. Ernaux choisit de transformer la vie de sa mère en une " histoire » pour se sentir «moins seule et factice dans le monde dominant des mots et des idées » (Ernaux, 1988, p. 106), D. Eribon mène le "projet [d']écrire sur le "retour" »(Eribon, 2018, p. 246) et de se confronter aux «fantômes de [s]on propre passé, qui surgirent dès après le décès " de son père (ibid., p.17). É. Louis (2021a, p.19), dans leur sillage, écrit "pour comprendre sa vie » [celle de sa mère], faire surgir "des fragments de vérité », et «faire jaillir des sentiments que le corps ne sait pas exprimer ».

13 Écrire sur leurs parents, c'est écrire sur leurs vies et donc écrire sur eux, les transfuges de classe. Lors de la journée d'étude le 2 octobre dernier à la Bibliothèque nationale de France, intitulée «Écrire sa vie, raconter la société : l'autobiographie au risque de la sociologie ", A. Ernaux et D. Eribon ont d'ailleurs rappelé avec insistance, qu'ils n'étaient pas des «transclasses" (Jacquet, 2015) - terme qui dépolitise ce dont il est question - mais bien des transfuges de classe qui ont fui la violence sociale, le terme «transfuges » rendant bien compte des difficultés à mettre en œuvre ce processus de fuite, des souffrances inhérentes et de l'idée de trahison que cela implique. En cherchant à reconstituer de l'intérieur le monde qu'ils ont fui, à reconstituer les codes et les valeurs de ce monde dont ils sont issus, sans être du côté dominant, sans aller contre ce monde, ils optent pour une écriture factuelle, et c'est un choix éminemment politique, qui n'est pas sans conséquence pour la réception de ces textes. En agissant de la sorte, en dessinant un je en jeu dans le monde qu'il habite, leur je intime s'universalise - A. Ernaux parle même de « je transpersonnel » (Ernaux, 1994) - ce qui permet la rencontre avec les lecteurs.

14 Cette rencontre s'inscrit en réalité dans une lignée de rencontres. Dès les deux premiers chapitres de Retour à Reims, D. Eribon cite tour à tour C. Simon, J. Genet, P. Nizan et A. Ernaux (Eribon, 2018, p. 12-13, 26-28) et affirme qu'il a « reconnu très précisément ce [qu'il] a vécu à ce moment-là en lisant les livres qu'Annie Ernaux a consacrés à ses parents et à la "distance de classe" qui la séparait d'eux » (ibid., p. 28)

Elle y évoque à merveille ce malaise que l'on ressent lorsqu'on revient chez ses parents après avoir quitté non seulement le domicile familial mais aussi la famille et le monde auxquels, malgré tout, on continue d'appartenir, et ce sentiment déroutant d'être à la fois chez soi et dans un univers étranger (Id.)

15 La reconnaissance se passe dans les mêmes termes pour É. Louis qui, à la lecture de Retour à Reims, a le sentiment de lire «l'histoire de [s]a vie » :

Je pense que c'est cette erreur, le fait que j'ai cru que c'était mon histoire, qui a fait que ce livre a eu autant d'impact sur moi. Je pense qu'il faut moins considérer les livres comme un espace à l'intérieur duquel on peut se reconnaitre que comme un espace de fantasme, et peut-être même se demander si ce n'est pas le vrai pouvoir qu'ont 
les grands livres, le fantasme, au sens d'une erreur dans l'identification qui produit des effets sur la réalité (Ibid., III).

Il n'y a pas - à mon sens - d'opposition stricte entre " reconnaissance » et " espace de fantasme ». Dans son Parcours de la reconnaissance, P. Ricœur montre en effet avec beaucoup de clarté pourquoi, lorsque l'on réfléchit à la question de l'identité, il s'agit toujours d'un « parcours » au sens d'un

passage de la reconnaissance-identification, où le sujet de pensée prétend effectivement à la maitrise du sens, à la reconnaissance mutuelle, où le sujet se place sous la tutelle d'une relation de réciprocité, en passant par la reconnaissance du soi dans la variété des capacités qui modulent sa puissance d'agir son agency (Ricœur, 2004, p. 359).

17 Ce "parcours de l'identité dans ses écarts » (ibid., p. 361) est dynamique et permet à D. Eribon de se reconnaitre dans A. Ernaux, et à É. Louis de se reconnaitre dans D. Eribon et dans A. Ernaux. C'est en réalité cette filiation elle-même qui est dynamique, les écrits produisant de nouveaux écrits : si A. Ernaux a beaucoup lu - de S. de Beauvoir, à P. Bourdieu en passant par C. Etcherelli -, « l'œuvre d'Annie Ernaux a [également] produit d'autres écrivains », comme cela a été rappelé au cours de la table ronde qui a clôturé la journée d'étude à la BNF ; dans les pays germanophones où l'écho aux textes de D. Eribon est très fort, on parle même aujourd'hui de "genre Eribon » lorsqu'il est question d'autosociobiographie ${ }^{6}$.

Ce «parcours de reconnaissance " n'est pas seulement celui d'écrivains; d'autres artistes ont été saisis par l'histoire et le je de ces auteurs: l'actualité cinématographique en est un marqueur fort. Après Retour à Forbach en 2017, R. Sauder vient notamment de réaliser un nouveau documentaire, J'ai aimé vivre là, sur les traces d'A. Ernaux dans la ville de Cergy Pontoise, sorti le 29 septembre $2021^{7}$. Mais c'est le théâtre qui en est l'illustration la plus emblématique. Chez ces metteurs en scène, le processus de reconnaissance fonctionne aussi à plein régime. L. Hatat rappelle dans le dossier de production de son spectacle qu'à l'âge de «dix-huit ans, [il a] quitté Reims, [s]a ville natale pour [s]e rendre à Paris en quête d'une vie de théâtre » (Hatat, 2014) ; les traces ne peuvent être plus transparentes. À des centaines de kilomètres de là, T. Ostermeier explique pourquoi il lui a paru évident de mettre en scène cet essai :

Ma première réaction immédiate a été le constat suivant : au moins, je ne suis pas seul. Car mon histoire - même si elle diffère à bien des égards - est similaire à celle d'Eribon, et que le sentiment d'avoir honte de ses origines m'est très familier. [...] Mais cette histoire - de quelqu'un qui essaie de quitter la province pour faire carrière dans le milieu intellectuel de la grande ville et qui a toujours honte de ses origines, n'en dit jamais un mot, mais essaie toujours de s'adapter à son nouveau milieu - je l'ai reconnue. Eribon se qualifie de «traitre de classe », et c'est aussi mon histoire (Pearson, 2017).

S. Nordey ne choisit pas de monter É. Louis parce qu'il reconnait sa propre histoire ; en revanche, il discerne immédiatement la force de son écriture et c'est ce qui le pousse, après une lecture d'Histoire de la violence au Théâtre national de Strasbourg, à lui proposer d'écrire pour le théâtre. Lorsqu'il reçoit le texte, Qui a tué mon père, il est immédiatement conquis :

J'ai aimé la simplicité et la force du texte, au carrefour de l'intime et du politique et, après une discussion avec Édouard, nous avons convenu que je porterai ce texte en l'interprétant et en le mettant en scène. Dans ma pratique d'acteur, l'une des lignes de force constituant mes choix est de porter les figures des auteurs eux-mêmes : je suis Falk Richter dans My Secret Garden et dans Je suis Fassbinder, je suis Christophe 
Pellet dans La Conférence, je suis Pascal Rambert dans Clôture de l'amour et dans Répétition, il est donc simple et évident pour moi de devenir É. Louis dans Qui a tué mon père. J'aime cette forme de consanguinité avec les auteurs, j'aime me glisser dans leur peau (Théâtre de la Colline, 2019, p. 7). monde et sa violence, le poussent à les interpréter sur le plateau. C'est également la poétique, la forme littéraire, qui attire l'attention d'I. van Hove, il reconnait dans la forme de Qui a tué mon père, la dimension monologuée à laquelle il s'est déjà confronté dans trois de ses précédentes créations (La voix humaine de J. Cocteau en 2009, Song from Far Away en 2015 et The other voice en 2016) :

Mettre en scène un monologue m'offre la possibilité de travailler intensivement et intimement avec un acteur de l'ensemble, au millimètre près. Tout comme mon père, un apothicaire à l'ancienne qui fabriquait encore ses propres pilules et onguents avec deux balances. Non en utilisant des kilogrammes mais avec des milligrammes, et chaque milligramme compte » (van Hove, 2020).

En s'inscrivant dans sa propre lignée familiale, en travaillant en mode microthéâtral et non en mode macrothéâtral, Ivo van Hove revient en quelque sorte au monde qui est le sien et donne à voir sur le plateau, comme son père dans son laboratoire, les rouages de l'infiniment petit qui portent l'histoire d'É. Louis.

Lorsque l'on fait retour sur les raisons qui poussent les metteurs en scène à porter les écritures de soi au plateau, on comprend mieux la force de la dimension performative de ces textes: en mettant en scène ces «énoncés performatifs, qui effectuent une action par le fait même d'être énoncés » (Pavis, 2018, p. 264), il devient possible de faire entendre et de faire vivre la réalité sociale qui est la leur, de faire résonner le «je transpersonnel » dans la salle de théâtre, car comme le signale Laurent Hatat : « Quand l'intime touche à l'universel » ce qui est le cas avec Retour à Reims ou avec Qui a tué mon père, car ces «livre [s]e frotte[nt] à l'universel », alors « le théâtre n'est qu'à un pas » (Hatat, 2014).

\section{De la puissance de l'incarnation aux performances transmédiales}

Que les metteurs en scène soient sensibles à la dimension autobiographique (L. Hatat et T. Ostermeier) et/ou à la dimension poétique (S. Nordey et I. van Hove), l'opération de transfert sur le plateau engage un processus d'éloignement, une distanciation du texte et donc de la parole des transfuges de classe et permet de déplier une réalité de plus en plus globale mais aussi de plus en plus intime, à la manière d'un documentaire.

\section{Retour à Reims}

Dans la mesure où le Retour de D. Eribon ne s'engage qu'au moment où sa mère vit seule après que son père a été placé dans une "clinique accueillant des personnes frappées par la maladie d'Alzheimer » (Eribon, 2018, p. 12) et ne sera rendu possible que par les multiples discussions qui vont se nouer alors entre la mère et le fils, L. Hatat choisit de "donn[er] une incarnation puissante de cet échange » (Hatat, 2014) en mettant en scène deux comédiens, A. Mathieu et S. Debrun. Pas question en revanche de s'inscrire dans un «naturalisme trop réducteur», il s'agit d'«affronter les mouvements d'une 
pensée en action » (Id.) : comment se comporter lorsqu'on a l'impression d'être à la fois complètement chez soi mais dans un monde étranger? Comment ne pas se mettre en colère face à l'aveuglement des siens "devant la violence du déterminisme social » (Id.) ? Ces questions sont au cœur du dialogue entre les deux acteurs mais également des monologues de chacun d'eux - qui le doublent en quelque sorte - pour mieux interroger chacun leur propre histoire, et, ce faisant, la mettent à distance. Ces questions naissent sur le plateau à l'endroit même où s'articulent «les corps des acteurs et l'omniprésence des images du passé » (Id.). La boite de photos familiales (dont il est souvent question dans Retour à Reims) est présente sur le plateau - par ailleurs très épuré - et donne l'occasion de revenir sur la culture ouvrière, la vie à l'usine, l'absence de joie au quotidien, tout en mettant en avant la logique de classe sociale qui permet de comprendre la construction du processus. Les deux acteurs jouent donc une sorte d'expérience sociologique qu'ils interrompent par moment pour interpeler le public, mais, alors que le spectacle «pourrait virer à la leçon sociologique simpliste et pesante, il n'en est rien. Grâce au talent des acteurs et d'un metteur en scène qui sait conjuguer l'intime et le politique » (Chevilley, 2016).

En 2017, T. Ostermeier propose sa propre version du Retour dans un dispositif plus complexe et complètement transmédial, dans lequel il part de la parole de D. Eribon et de son vécu pour finir par déborder dans le présent à travers les discussions des personnages, de sorte que les spectateurs sont alors en mesure d'engager un dialogue avec leur propre histoire. Sur le plateau, c'est le décor d'un studio d'enregistrement qui se voit reconstitué. Une actrice, Katy/Catherine (en fonction des versions), enregistre la voix-off pour le film documentaire du livre de D. Eribon, Retour à Reims, sous la direction de son ami réalisateur, Paul. Le cadre dramaturgique est posé. La première partie du spectacle est consacrée à l'enregistrement de la voix-off - un montage du texte opéré par Paul - pour le film, projeté sur grand écran en fond de scène. Ce qui pourrait apparaitre de prime abord comme une illustration visuelle et scénique du livre se révèle bien plus une mise en perspective de ce texte, première étape de la distanciation orchestrée sur scène. En effet, Irène Jacob, la voix-off française, le souligne :

La pensée d'Eribon ne doit pas s'imposer mais se proposer au spectateur comme le déroulement d'une réflexion intime et sociologique. Cette pensée se découvre à l'instant même où elle se prononce, ce n'est pas un prêche. Thomas m'a donné à lire l'essai de Kleist, De l'élaboration progressive des idées par la parole, qui parle du processus du discours et de l'écoute : on peut développer une pensée parce que l'écoute de quelqu'un permet de poser des mots, d'être face à ses interrogations et d'avancer dans la réflexion (TNS, 2019a, p. 9).

Le film documentaire s'ouvre sur des images en gros plan d'un Eribon visiblement absorbé par ses pensées dans le TGV qui relie Paris à Reims. Pendant près de quarantecinq minutes, ce sont toutes sortes d'images que va faire revivre ce documentaire. Des prises de vue de Reims et de sa banlieue aujourd'hui côtoient des images d'archives; D. Eribon apparait avec sa mère, chez elle - une idée que le metteur en scène a d'abord eu du mal à faire accepter au sociologue mais qui finit par s'y ranger et dont il rappelle aujourd'hui encore, combien cela l'a «bouleversé, parce que l'image apporte quelque chose que l'écriture ne peut pas apporter ${ }^{9}$ »-puis dans son quotidien parisien; un extrait d'Apostrophe de B. Pivot, des images du clip de F. Hardy « Tous les garçons et les filles de mon âge » et celles aussi du film La Belle et la Bête avec J. Marais dans le rôletitre. 
27 À l'issue de la première partie du spectacle, Katy commence à interroger les choix de son ami, les coupes qu'il opère dans le texte, la sélection et le montage des images, l'adéquation du texte de la voix-off et des images projetées - elle n'accepte pas par exemple la présence d'images de gilets jaunes qui semblent être assimilés à des militants d'extrême-droite - et c'est une véritable discussion politique qui s'engage sur le plateau : Que peut-on vraiment dire? Quelles sont les forces politiques à l'œuvre ? Quelle est la responsabilité de la gauche dans la montée de l'extrême-droite ? Peut-on remettre efficacement le système en question?

Dans la deuxième partie, la partition dramaturgique se trouve de plus en plus prise en charge par les personnages qui apparaissent comme des prolongements de D. Eribon : en replongeant chacun dans leur propre histoire et en conjuguant leur histoire individuelle à l'Histoire collective, les personnages nous offrent une nouvelle lecture de la violence sociale, un véritable miroir de notre temps dirait $\mathrm{W}$. Shakespeare. Paul choisit alors d'intégrer ces témoignages à son film et de prolonger ainsi la réflexion politique de D. Eribon. Et c'est là que la multiplicité des versions de Retour à Reims prend tout son sens, car, à chaque fois, une partie du texte a été écrite à partir de l'histoire des participants, en prenant la couleur du pays en question.

Dans les versions anglaise et allemande, le passé de N. Hoss rencontre celui de la comédienne qu'elle incarne lorsqu'elle évoque son propre père: issu d'une famille ouvrière, de père communiste, témoin dès son enfance de la violence sociale, il s'engage toute sa vie dans des luttes très concrètes. Après avoir rejoint le parti communiste, il étudie la philosophie puis s'établit à Stuttgart où il fonde un syndicat indépendant, crée le parti vert allemand qu'il finit par quitter sur fond de désaccord majeur. Il s'investit alors dans l'aide aux peuples d'Amazonie et c'est sur ces images de W. Hoss au cœur de la forêt amazonienne, projetées via le portable de Katy, que s'achève le spectacle : les trois comédiens, réunis au centre du plateau, fixent le portable, un halo de lumière tamisée les enveloppe et recrée l'intimité autour de la figure du père qui déborde du cadre initial, comme si le père de D. Eribon, puis le père de Katy devenaient des figures de transfert universelles.

Dans la version française, c'est Blade Mc Alimbaye (Tony), artiste musicien d'origine normande et d'ascendance sénégalaise, qui prend en charge la narration en racontant l'histoire de son grand-père, ancien tirailleur sénégalais, immigré de la première génération, rescapé du massacre de Thiaroye, autre écho terrible de la violence sociale. Tony prend la place de Catherine au pupitre, et alors qu'ils regardent ensemble la dernière photo du grand-père, les premières notes du morceau « Février noir » issu de l'album Bleu : point zéro (2015) résonnent: "J'ai la peau corbeau, m'a fait savoir l'administration / Radote l'intégration, je les attends depuis trois générations / Grandpère, la chair à canon, les y’a bon, les jours précaires / Souviens-toi, Le Havre était une ville négrière ».

31 Dans la version italienne, c'est également un rappeur d'origine nigériane qui prend le relais au micro et dans la nouvelle version allemande, c'est l'histoire du père d'I. Redfern qui sert de fil conducteur à l'épilogue du spectacle, un Noir américain, recruté par la Navy, stationné en Allemagne. I. Redfern raconte son expérience du racisme aux États-Unis et en Allemagne, engageant un dialogue avec Amewu Nowe, un rappeur noir berlinois.

32 Dans la récente version suédoise, L. Endre, l'actrice qui joue Katy, «parle de ses racines ouvrières $^{10} »$ (Lundström, 2021). Par ailleurs, lors d'une discussion organisée par le 
Théâtre national de Suède (Dramaten) à Stockholm le 15 octobre dernier, les trois acteurs - L. Endre, P. Pazooki et J. Söderblom - ont pu revenir sur les enjeux d'être à la fois sur scène et d'être soi-même, puisqu'ils mettent leur histoire personnelle au service de la création d'une œuvre théâtrale qui s'ancre dans une histoire, celle de l'Europe en général, mais aussi celle de la Suède aujourd'hui. La critique ne s'y est pas trompée et a relevé qu'au travers de ce dispositif, "Thomas Ostermeier crée une nouvelle forme hybride de théatre politique - un semi-documentaire qui offre des perspectives sur des questions d'actualité ${ }^{11}$ » (Ring, 2021). En réalité, non seulement ils documentent l'actualité mais, en performant le réel, ils documentent aussi l'intime.

\section{Qui a tué mon père}

Dans ce texte, É. Louis revient vers son père qu'il n'a pas revu depuis des années et découvre un corps qu'il reconnait à peine, souffrant, lourd, impotent. C'est là qu'il se décide à écrire une nouvelle "histoire", celle du corps de son père. C'est donc une «biographie par le prisme de [la] relation » (TNS, 2019b, p. 13) entre son père et lui, à laquelle il choisit de donner une dimension très politique puisque ce texte accuse nominativement (Louis, 2018a, p. 75-84) les politiques qui ont tué son père : «L'histoire de ton corps accuse l'histoire politique " (ibid., p. 84). Comparer la matérialisation scénique du père absent dans les mises en scène de S. Nordey, de I. van Hove et de T. Ostermeier apparait très fructueux pour appréhender la manière dont la performance s'articule au théâtre afin de développer une nouvelle approche du réel. Que faire en effet de ce père intensément spectral ? Fantôme dans la vie (quand il n'est pas absent, son fils «espère son absence » (ibid., p. 14), fantôme dans l'écriture (il ne parle pas ou seulement à travers son fils), comment présentifier l'absence sur scène ?

Souvenons-nous du préambule d'É. Louis (2018a, p. 9) :

Si ce texte était un texte de théâtre, c'est avec ces mots-là qu'il faudrait commencer : un père et un fils sont à quelques mètres l'un de l'autre dans un grand espace vide. Cet espace pourrait être un champ de blé, une usine désaffectée et déserte, le gymnase plastifié d'une école. Peut-être qu'il neige. Peut-être que la neige les recouvre petit à petit jusqu'à les faire disparaitre.

C'est exactement ce que S. Nordey nous donne à voir sur le plateau : il est seul dans un immense espace vide. Il arpente deux heures durant un plateau rectangulaire qui change par moment de couleur. Au centre, une table et deux chaises. Sur le fond de scène est projetée une photo en noir et blanc: celle des toits d'un village qui pourrait bien être Hallencourt, le village dont É. Louis est originaire ou celui où vit ce père aujourd'hui. Parfois, la photo est remplacée par des extraits du texte. Et puis, il neige aussi par moment. Pour figurer le père absent, l'idée première a été de matérialiser le père par un corps vivant, celui d'un acteur, mais très vite cette équivalence est apparue "fausse et réductrice» (TNS, 2019b, p. 40). Puis a surgi l'idée de proposer au père d'É. Louis de monter sur scène, mais cela s'est avéré impossible. "La présence de JeanPierre Mocky ", le propre père de S. Nordey a même été envisagée, "mais cela aurait donné à la pièce un autre sens" (Nordey, 2020). Il faut pourtant bien parler à quelqu'un, adresser le texte à cet autre. C'est l'expérimentation avec un mannequin qui a fini par faire sens dans ce spectacle. De cette présentification naissent entre les mains d'A. Leray, artiste sculptrice, plusieurs mannequins, plusieurs pères, des pères aux postures différentes qui « racontent les symptômes d'une vie» (TNS, 2019b, p. 41). La fragmentation du père en plusieurs corps engage une forme d'universalisation du 
récit: ce n'est plus un seul père dont on raconte l'histoire, cela devient l'histoire de tous ces pères qui, comme celui d'É. Louis sont restés des invisibles tout au long de leur vie, des pères absents de l'histoire familiale mais aussi de l'histoire sociale et politique. S. Nordey, tour à tour jeune garçon, adolescent et homme adulte, est un enfant « crédible, parce que la scène, c'est un pacte passé avec le spectateur » (Nordey, 2020), un enfant de tout âge, qui cherche à retrouver les émotions de son enfance en les performant, seul en scène, et après s'être "démené comme un beau diable " sur le plateau, « au salut, il est comme ravagé. C'est beau un acteur ravagé par le texte qu'il vient de dire » (Thibaudat, 2019).

I. van Hove choisit, de son côté, de traduire et d'adapter le texte d'É. Louis pour l'un des grands acteurs de sa troupe, H. Kesting et il en offre une performance tout à fait magistrale, saluée par la critique. Dans un décor très marqué socialement - les murs sombres de la pièce étroite sont délabrés, pleins des trous que créent les coups de poing du père, l'ameublement est spartiate - on ne trouve qu'un vieux lit à ressorts et un téléviseur d'un autre âge, évoquant une vie misérable qui fait écho à celle décrite dans le livre, sans qu'il soit possible de déterminer si l'action se joue aujourd'hui où à l'époque de l'enfance d'É. Louis. Hans Kesting porte le texte seul pendant près d'une heure et demie, et, tout au long de sa performance, il donne vie au père et au fils, passe de l'un à l'autre sans peine, jouant tour à tour le vieil homme malade et le jeune garçon qui a besoin de l'attention de son père, dressant des portraits impitoyables et émouvants : lorsqu'il joue le père, il courbe le dos et glisse ses mains dans son pull, figurant un ventre lourd et affaissé ; par moment, il ouvre la porte en fond de scène, laissant entrer une lumière sourde ${ }^{12}$, et allume une cigarette, on entend alors ses poumons qui sifflent et les quintes de toux se répètent inlassablement. Toujours sur le seuil, il ne peut pas sortir de là où il est, de ce qu'il est. À d'autres moments, l'espace scénique est plein d'une épaisse fumée, comme pour figurer l'oppression, l'impossibilité de respirer dans ce milieu social qui ne lui laissera aucune chance; $\mathrm{H}$. Kesting alterne entre rage et désespoir et semble ne pas pouvoir sortir de ce huisclos qui le fait visiblement atrocement souffrir. La scène où Édouard enfant regarde le film Titanic sur l'écran de télévision est particulièrement bouleversante : H. Kesting chante d'abord la chanson de $\mathrm{C}$. Dion a capella, puis assis à même le sol, il écarte les bras - comme L. DiCaprio et K. Winslet dans le film - pour essayer de sentir le vent, la liberté, il rêve de s'envoler, mais cela n'est pas pour lui, seules les notes de musique s'envolent. Ce que l'on retient de cette mise en scène, c'est indéniablement la performance scénique bouleversante de H. Kesting qui parvient à incarner alternativement avec brio des personnages si contrastés et des sentiments si forts, de sorte qu'il opère une liaison entre le père et le fils qui deviennent donc consubstantiels : en allant au-delà des faits, il montre le lien indiscutable qui unit le père et le fils, malgré tout, performant ici une véritable relation universelle.

Chez T. Ostermeier, la proposition scénique est toute autre. É. Louis incarne son propre rôle, il est donc performeur, "celui qui parle et agit en son nom propre (en tant qu'artiste et personne) et s'adresse ainsi au public », de sorte que « le performer effectue une mise en scène de son propre moi », là où " l'acteur joue le rôle d'un autre » (Pavis, 2019, p. 395). Le père n'est pas absent, mais c'est un vrai fantôme, à la fois absent et présent: invisible, assis dans un fauteuil, il dispose d'une couverture posée sur le dossier. Dans ce spectacle, c'est le corps de l'acteur-performeur qui sert la présentification de l'absence, le corps étant compris au sens de la chair, en tant que 
substance considérée principalement dans sa réalité profonde: É. Louis incarne ici, avec sa chair, son propre corps et celui de son père. Tous ses gestes, toutes ses adresses tendent vers le père absent: assis au bureau, il lui jette des regards d'une forte expressivité ; debout à côté du fauteuil, une forme de tendresse se manifeste, «parfois leurs peaux se touchent, ils entrent en contact mais, même-là, même dans ces moments-là, ils restent absents l'un de l'autre » (Louis, 2018, p. 10) ; à un moment, il s'assoit même dans le fauteuil, s'enroule dans la couverture comme dans une seconde peau, celle de son père ; enfin, toutes les chorégraphies musicales au travers desquelles il revit son enfance sont adressées à l'éternel absent; en chantant, en dansant, en s'épuisant physiquement, son corps s'affirme "comme un producteur d'intensité et d'énergie sans unités identifiables », interrogeant ainsi « les procédés d'intensification et de stylisation sur les affects et leur impact sur le spectateur » (Pavis, 2018, p. 80). Dès l'acte d'écriture, É. Louis (2018b, p. 5) avait noté que «c'est cette conscience qui [lui] permet aujourd'hui seulement de vivre les moments de joie de [s]on enfance ». Comme si le fait de re-jouer son histoire avec ce décalage spatial et temporel permettait une réincarnation : c'est à la fois le même corps, celui d'É. Louis, mais ce n'est pourtant plus le même corps puisqu'il re-vit une expérience; dans la performance, il aura probablement découvert une épaisseur de vie supplémentaire, ce que la journaliste du New York Times présente dans la salle n'a pas manqué de relever, sa performance révélant " un véritable instinct et une intuition de la scène authentique » tout en signalant que " ce serait injuste de dire qu'il était plus crédible que [...] Stanislas Nordey ${ }^{13}$ " (Cappelle, 2020). On le voit bien, le théâtre n'en a toujours pas fini de questionner notre rapport à l'identité.

\section{Conclusion}

L'écriture de soi, que d'aucuns considèrent comme profondément intime et personnelle, permet pourtant aux transfuges de classe de faire vivre un je ancré dans le réel social et politique, un je qui peut devenir un autre je et même un nous. Les metteurs en scène ne s'y sont pas trompés et ont pleinement reconnu le potentiel théâtral qu'offrent ces écrits. Recréés pour la scène, au travers d'un processus de dramatisation, ils permettent d'ouvrir le plateau à des pans du réel sans renoncer à la littérature. Au croisement du théâtre et de la performance, ces spectacles, à l'exception de celui de et par É. Louis - même si cela reste discutable dans la mesure où il vient de jouer plusieurs soirs de suite Qui a tué mon père à Berlin, re-présentant ainsi chaque soir le spectacle prennent appui sur un texte non-dramatique et ne sont ni des happenings, ni des actions spectaculaires, ni des expériences théâtrales, mais bien des représentations théâtrales. Et pourtant, nous l'avons vu, il est indispensable de «tenir compte de leur possible performance scénique » (Pavis, 2018, p. 264-265). Dès que l'acteur apparait sur le plateau,

il est en quelque sorte mis dans un cadre sémiotique et esthétique qui l'utilise dans l'univers dramatique fictif. Toutes ses propriétés physiques (sa beauté, sa sexualité, son "être mystérieux») sont sémiotisées, transférées sur le personnage qu'il représente [...]. [Même si] ceci ne veut pas dire que nous ne pouvons pas percevoir directement ce comédien comme un être humain, qui existe comme nous, et que nous pouvons désirer (Pavis, 2019, p. 457). transmédiales où le théâtre et la réalité se croisent de manière si belle et si intelligente 
qu'il n'est plus interdit de rêver : Le théâtre est bien le lieu privilégié où les chemins se croisent de manière dynamique, où des réseaux de sens se créent, avec le souci d'adopter « une attitude militante de recherche d'un tout d'une complexité fascinante " (Claverie, 2010, p. 23).

\section{BIBLIOGRAPHIE}

CAPPELle, L. (2020). «Star Directors Pull Back the Curtain on How They Work ». New York Time. En ligne : https://www.nytimes.com/2020/02/20/theater/peter-brook-paris-thomas-ostermeieredouard-louis.html

CHeVILley, P. (2015). « Retour à Reims : petite leçon de théâtre politique. Libération. En ligne : https://www.lesechos.fr/2015/02/retour-a-reims-petite-lecon-de-theatre-politique-243077 CLAVERIE, B. (2010). «Pluri-, Inter-, Transdisciplinarité : ou le réel décomposé en réseaux de savoir », projectics, Proyéctica, Projectique 2010/1, n 4, p. 5-27.

DuUIS, J. (2009). « Autobiographie. L'intello d'en bas ». L'Express, 3043, p. 107.

ERIBON, D. (2018) [2009]. Retour à Reims, Paris : Flammarion.

ERnaUX, A. (2009). « Fils de la honte ». Le Nouvel Observateur, 22 oct., p. 118.

ERNAUX, A. (1994). «Vers un je transpersonnel ». Recherches interdisciplinaires sur les textes modernes, 6, p. 218-221.

ERnauX, A. (1988). Une Femme. Paris : Gallimard.

FERNIOT, C. (2009). « D. Eribon - Retour à Reims ». Télérama, 3122, p. 69.

HATAT, L. (2014). « Dossier de production ». Animamotrix. En ligne : https://7dc84abd-b6b3-468aa001-bffof1524e4d.filesusr.com/ugd/33aaeb_c1045a74065f40d3845c8f386cb8f650.pdf

Hove VAN, I. (2020). « Program Brochure ». Internationaal Theater Amsterdam. En ligne : https:// ita.nl/en/shows/wie-heeft-mijn-vader-vermoord/1654756/

JACQUET, C. (2015) [2014]. Les Transclasses ou la non-reproduction. Paris : Presses universitaires de France.

JEANNELLE, J.-L. (2009). « "Retour à Reims" de D. Eribon : D. Eribon, du "verdict sexuel” à la "honte sociale" ». Le Monde. En ligne : https://www.lemonde.fr/livres/article/2009/10/29/retour-areims-de-didier-eribon_1260093_3260.html

LADOUCEUR, B. (2010). « D. Eribon, Retour à Reims ». Lectures. Les comptes rendus. En ligne : http:// journals.openedition.org/lectures/898

LEJEUNE, P. (1996) [1975]. Le Pacte autobiographique. Paris : Éd. Le Seuil.

LEPRINCE, C. (2019). « Comment Retour à Reims est devenu un best-seller sociologique ». France Culture. En ligne : https://www.franceculture.fr/sociologie/comment-retour-a-reims-est-devenuun-best-seller-sociologique 
LouIs, É. (2018a). Qui a tué mon père. Paris : Éd. Le Seuil.

LouIs, É. (2018b). Propos recueillis par Fanny Mentré pour le Théâtre National de Strasbourg. En ligne : https://www.theatre-contemporain.net/images/upload/pdf/f-f29-5cd3c78a85a00.pdf

LouIs, É. (2021a). Combats et Métamorphoses d’une femme. Paris : Éd. Le Seuil.

LouIs, É. (2021b). « En conversation avec D. Eribon », Séminaire Les mondes de la littérature par

D. Eribon à l'École Polytechnique de Zurich. En ligne : https://main.podigee-cdn.net/media/

podcast_27619_gesstebuch_livre_d_or_libro_degli_ospiti_episode_437188_edouard_louis_en_conversation_avec_didier_eribon.mp3? $\mathrm{v}=1620118628 \&$ source $=$ webplayer-download

LOUIS, É. \& OSTERMEIER, T. (2019). Au cœur de la violence. Paris : Éd. Le Seuil.

LUNDSTRÖM, J. (2021). « “Tillbaka till Reim” är ett navelskådande som angår oss alla », Dagens

Nyheter. En ligne : https://www.dn.se/kultur/tillbaka-till-reims-ar-ett-navelskadande-som-

angar-oss-alla/

NoRDEY, S. (2020). « “Je joue dans la joie, même les rôles les plus durs”. Entretien avec Alexandre

Demidoff ». Le Temps. En ligne : https://www.letemps.ch/culture/stanislas-nordey-joue-joie-

meme-roles-plus-durs)

PAVIS, P. (2018). Dictionnaire de la performance et du théâtre contemporain, Malakoff : A. Colin.

PAVIS, P. (2019) [1980]. Dictionnaire du théâtre. Paris : A. Colin.

PEARSON, J. (2017). « Klassenverräter. D. Eribon und Thomas Ostermeiers Rückkehr nach Reims ». Schaubühne. En ligne : https://www.schaubuehne.de/de/blog/klassenverraeter-brdidier-eribonund-thomas-ostermeiers-rueckkehr-nach-reims.html

RICeUUR, P. (2004). Parcours de la reconnaissance. Paris : Éd. Stock.

RING, L. (2021). «Tillbaka till Reims Lågmäld Lena Endre i ny slags politisk teater », Svenska

Dagbladet. En ligne : https://www.svd.se/lagmald-lena-endre-i-ny-slags-politisk-teater

THÉÂTRE DE LA COLLINE (2019). « Dossier de presse sur Qui a tué mon père ». Théâtre de la Colline. En

ligne : https://www.colline.fr/sites/default/files/dp-qui-a-tue.pdf

THÉÂTRE NATIONAL De STRASBouRg (2019a). « Retour à Reims de D. Eribon. Programme. Saison 19-20 ». En ligne : https://www.tns.fr/sites/default/files/document/retour_a_reims_-_prog.pdf

THÉÂtRe NATIONAL DE STRASBourg (2019b). «Qui a tué mon père d'É. Louis. Programme. Saison 19-20 ». En ligne : https://www.tns.fr/sites/default/files/document/qui_a_tue_mon_pere_-

_prog_all_bd.pdf

THIBAUDAT, J.-P. (2019). «Stanislas Nordey, un acteur textocentrique ». Balagan, le blog de Jean-Pierre

Thibaudat. En ligne : https://blogs.mediapart.fr/jean-pierre-thibaudat/blog/280319/stanislas-

nordey-un-acteur-textocentrique

\section{NOTES}

1. On doit le terme " autosociobiographie » à A. Ernaux - comme elle l'a rappelé lors de la journée d'étude "Écrire sa vie, raconter la société. L'autobiographie au risque de la sociologie » organisée par la Bibliothèque nationale de France le 2 octobre 2021 - afin de pouvoir « dire ce qu'elle avait fait avec La Place» (1983). 
2. En raison de la pandémie de Covid-19, la première de ce spectacle a finalement eu lieu le 7 octobre 2021 dans le cadre du Festival FIND 2021 à la Schaubühne.

3. Je souligne.

4. Date de la première dans le cadre du Festival FIND 2021.

5. Dont j'ai eu le plaisir de faire partie.

6. Voir à ce sujet E. Kargl et B. Terrisse (dirs), «Transfuge, transfert, traduction : la réception de D. Eribon dans les pays germanophones ", Lendemains 45 (180), Décembre 2020 (paru le 27 septembre 2021).

7. Par ailleurs, déjà le 11 août 2021, on découvrait au cinéma Passion simple de D. Arbid, alors que le 24 novembre prochain sortira le film d'A. Diwan, L'événement, tous deux tirés des textes éponymes d'A. Ernaux.

8. "Directing a monologue offers me the possibility to work intensively and intimately with one actor from the ensemble down to a fraction of an inch. Just like my father, an old fashioned apothecary who still made his own pills and ointments with a pair of scales. Not with kilograms but with milligrams, and each milligram count », ma traduction.

9. Propos de D. Eribon lors de son entretien avec L. Jung à la BNF le 2 octobre 2021.

10. «Lena Endre pratar om sina arbetarklassrötter », ma traduction.

11. «Tyske regissören Thomas Ostermeier skapar en ny hybridform av politisk teater - en semidokumentär som ger perspektiv på aktuella frågor », ma traduction.

12. J'emprunte cette expression à Bernard-Marie Koltès dans L'Héritage, Paris, Éd. Minuit, 2014, p. 49.

13. "with genuine instinct and feeling" - "it might be unfair to say that he was far more believable than the actor and director who initially commissioned and performed "Who Killed My Father" for the stage, Stanislas Nordey ", ma traduction.

\section{RÉSUMÉS}

Cette étude sur l'autosociobiographie en scène vise à analyser les raisons qui poussent de grands metteurs en scène européens à s'emparer des textes des transfuges de classe pour les porter au plateau et les choix artistiques qu'ils mettent en œuvre. L'écriture de soi dans sa dimension performative fait apparaitre un «je transpersonnel» qui permet de dépasser les dichotomies réalité/fiction, texte/performance, texte dramatique/texte non-dramatique et ouvre le plateau à des pans entiers du réel. Qu'il s'agisse d'incarner ces textes ou d'en faire de véritables performances transmédiales, l'écriture de soi au plateau permet à la fois de performer le réel et de documenter l'intime, en tant qu'elle est à la croisée d'enjeux esthétiques et politiques. L'étude s'appuie notamment sur Retour à Reims de D. Eribon et Qui a tué mon père d'É. Louis.

Abstract : This study on autosociobiography on stage aims at analyzing the reasons that urge great European directors to focus on the texts of class defectors to bring them to the stage and the artistic choices they implement. The writing of oneself in its performative dimension brings to light a "transpersonal I" that allows us to go beyond the reality/fiction, text/performance, 
dramatic text/non-dramatic text dichotomies, and opens the stage to whole sections of reality. Whether the approach consists in embodying these texts or making them into true transmedia performances, writing oneself on the stage makes it possible to both perform the real and document the intimate, insofar as it is at the crossroads of aesthetic and political issues. This study particularly rests on D. Eribon's Retour à Reims and É. Louis' Qui a tué mon père.

\section{INDEX}

Mots-clés : autosociobiographie, réel, performance, représentation, transfuges, D. Eribon, É. Louis

Keywords : autosociobiography, reality, performance, representation, class-defectors, D. Eribon, É. Louis

\section{AUTEUR}

\section{DELPHINE EDY}

Université de Strasbourg, Accra, F-67081 Strasbourg, FranceSorbonne Université, CRLC, F-75006 Paris, France 